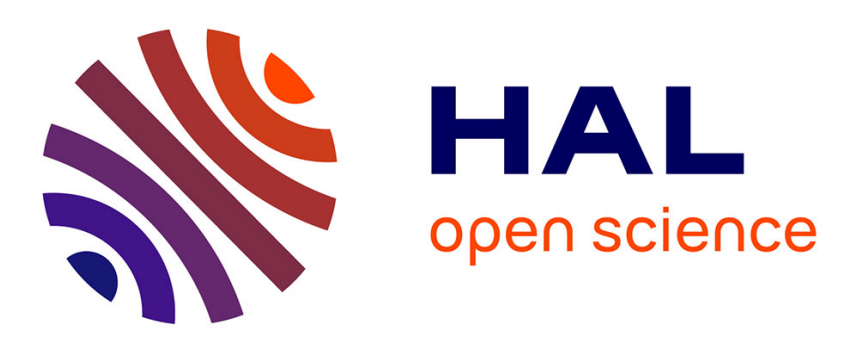

\title{
A Syntactical Approach to Qualitative Constraint Networks Merging
}

Jean-Francois Condotta, Souhila Kaci, Pierre Marquis, Nicolas Schwind

\section{To cite this version:}

Jean-Francois Condotta, Souhila Kaci, Pierre Marquis, Nicolas Schwind. A Syntactical Approach to Qualitative Constraint Networks Merging. LPAR: Logic for Programming, Artificial Intelligence and Reasoning, Oct 2010, Yogyakarta, Indonesia. pp.233-247, 10.1007/978-3-642-16242-8_17 . hal00866877

\section{HAL Id: hal-00866877 https://hal.science/hal-00866877}

Submitted on 23 Sep 2019

HAL is a multi-disciplinary open access archive for the deposit and dissemination of scientific research documents, whether they are published or not. The documents may come from teaching and research institutions in France or abroad, or from public or private research centers.
L'archive ouverte pluridisciplinaire HAL, est destinée au dépôt et à la diffusion de documents scientifiques de niveau recherche, publiés ou non, émanant des établissements d'enseignement et de recherche français ou étrangers, des laboratoires publics ou privés. 


\title{
A Syntactical Approach to Qualitative Constraint Networks Merging
}

\author{
Jean-François Condotta, Souhila Kaci, Pierre Marquis and Nicolas Schwind \\ Université Lille-Nord de France, Artois, F-62307 Lens \\ CRIL, F-62307 Lens \\ CNRS UMR 8188, F-62307 Lens \\ \{condotta, kaci, marquis, schwind\}@cril.univ-artois.fr
}

\begin{abstract}
We address the problem of merging qualitative constraint networks (QCNs) representing agents local preferences or beliefs on the relative position of spatial or temporal entities. Two classes of merging operators which, given a set of input QCNs defined on the same qualitative formalism, return a set of qualitative configurations representing a global view of these QCNs, are pointed out. These operators are based on local distances and aggregation functions. In contrast to QCN merging operators recently proposed in the literature, they take account for each constraint from the input QCNs within the merging process. Doing so, inconsistent QCNs do not need to be discarded at start, hence agents reporting locally consistent, yet globally inconsistent pieces of information (due to limited rationality) can be taken into consideration.
\end{abstract}

\section{Introduction}

Qualitative representation of time and space arises in many domains of Artificial Intelligence such as language processing, computer vision, planning. One needs to take advantage of a qualitative formalism when the available information about a set of spatial or temporal entities is expressed in terms of non-numerical relationships between these entities (e.g., when information comes primarily from natural language sentence). Starting from Allen's formalism [1] basically used to represent relative positions of temporal intervals, many other qualitative formalisms have been put forward in the literature these last three decades $[24,19$, $15,2,8,20]$. Besides temporal and spatial aspects, these formalisms also constitute powerful representation settings for a number of applications of Artificial Intelligence, such as reasoning about preferences [9] or multiple taxonomies [23].

When we are asked to express a set of preferred or believed relationships between entities, we are generally more willing to provide local relations about a small number of entities from which the underlying set of preferred or possible configurations about the whole set of entities can be deduced. Consider for example a student, William, who expresses his preferences on the schedule of four courses (Operating Systems, Algebra, Analysis, Programming). William prefers to learn Analysis after Algebra. Assume William would also like to learn 
Programming after Analysis and wants to start learning Programming before Algebra finishes. Then no schedule can satisfy all his preferences, since satisfying two of his wishes implies the third one to be discarded. Obviously, conflicts can also arise in the case when several students are asked to express their preferences on a common schedule.

In this paper we address the problem where several agents express their preferences / beliefs on relative positions of (spatial or temporal) entities. This information is represented, for each agent, by means of a qualitative constraint network (QCN). A procedure for merging QCN has been proposed in [6], directly adapted from a "model-based" method for merging propositional knowledge bases $[13,14]$. This procedure is generic in the sense that it does not depend on a specific qualitative formalism. It consists in defining a merging operator which associates with a finite set of QCNs a set of consistent (spatial or temporal) information representing a global view of the input QCNs. While this method represents a starting point in the problem of merging QCNs, it has however some limitations. First, a QCN to be merged is reduced to its global possible configurations; therefore inconsistent QCNs are discarded. As we will show in the paper, even if a QCN is inconsistent, it may however contain relevant information which deserves to be considered in the merging process. Secondly, this approach is expensive from a computational point of view as it requires the computation of all possible configurations about the whole set of entities. This paper aims at overcoming the above limitations. We propose a syntactical approach for merging QCNs in which each constraint from the input QCNs participates in the merging process. We define two classes of QCN merging operators, where each operator associates with a finite set of QCNs defined on the same qualitative formalism and the same set of entities a set of consistent qualitative configurations representing a global view of the input set of QCNs. Each operator is based on distances between relations of the underlying qualitative formalism and on two aggregation functions.

The rest of the paper is organized as follows. The next section recalls necessary preliminaries on qualitative constraint networks, distances between relations of a qualitative formalim and aggregation functions. In Section 3, we address the problem of dealing with conflicting QCNs. We introduce a running example and give some postulates that QCN merging operators are expected to satisfy. In Section 4, we define the two proposed classes of QCN merging operators and discuss their logical properties. We give some hints to choose a QCN merging operator in Section 5, and also give some comparisons with related works. We conclude in the last section and present some perspectives for further research. The proofs are in the Appendix.

\section{Preliminaries}

\subsection{Qualitative formalisms and qualitative constraint networks}

A qualitative formalism considers a finite set $B$ of basic binary relations defined on a domain $\mathrm{D}$. The elements of $\mathrm{D}$ represent the considered (spatial or tempo- 
ral) entities. Each basic relation $b \in \mathrm{B}$ represents a particular relative position between two elements of $D$. The set $B$ is required to be a partition scheme [16], i.e., it satisfies the following properties: (i) $B$ forms a partition of $D \times D$, namely any pair of $D \times D$ satisfies one and only one basic relation of $B$; (ii) the identity relation on $\mathrm{D}$, denoted by $e q$, belongs to $\mathrm{B}$; lastly, (iii) if $b$ is a basic relation of $\mathrm{B}$, then its converse, denoted by $b^{-1}$, also belongs to $\mathrm{B}$.

For illustration we consider a well-known qualitative formalism introduced by Allen, called Interval Algebra [1]. This formalism considers a set $\mathrm{B}_{\text {int }}$ of thirteen basic relations defined on the domain of non-punctual (durative) intervals over the rational numbers: $\mathrm{D}_{\text {int }}=\left\{\left(x^{-}, x^{+}\right) \in \mathbb{Q} \times \mathbb{Q}: x^{-}<x^{+}\right\}$. An interval typically represents a temporal entity. The basic relations of $\mathrm{B}_{\text {int }}=$ $\{e q, p, p i, m, m i, o, o i, s, s i, d, d i, f, f i\}$ are depicted in Figure 1. Each one of them represents a particular situation between two intervals. For example, the relation $m=\left\{\left(\left(x^{-}, x^{+}\right),\left(y^{-}, y^{+}\right)\right) \in \mathrm{D}_{\text {int }} \times \mathrm{D}_{\text {int }}: x^{+}=y^{-}\right\}$represents the case where the upper bound of the first interval and the lower bound of the second one coincide.

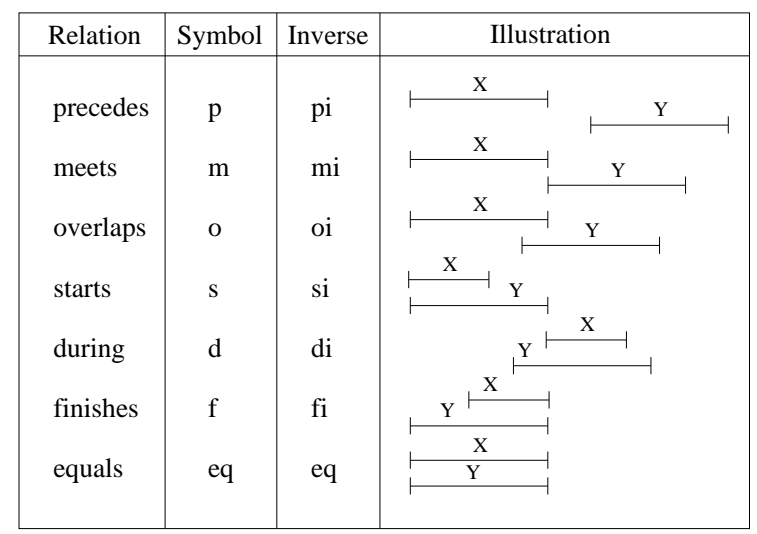

Fig. 1. The basic relations of Interval Algebra.

Given a set B of basic relations, a complex relation is the union of basic relations and is represented by the set of the basic relations it contains. In the following we omit the qualifier "complex". For instance, considering Interval Algebra, the set $\{m, d\}$ represents the union of the basic relations $m$ and $d$. The set of all relations is denoted by $2^{\mathrm{B}}$.

Pieces of information about the relative positions of a set of (spatial or temporal) entities can be represented by means of qualitative constraint networks (QCNs for short). Formally, a QCN (on B) is defined as follows:

Definition 1 (Qualitative constraint network). $A$ QCN $N$ is a pair $(V, C)$ where:

$-V=\left\{v_{1}, \ldots, v_{n}\right\}$ is a finite set of variables representing the entities, 
- $C$ is a mapping which associates with each pair of variables $\left(v_{i}, v_{j}\right)$ a relation $N[i, j]$ of $2^{\mathrm{B}}$. C is such that $N[i, i]=\{e q\}$ and $N[i, j]=N[j, i]^{-1}$ for every pair of variables $v_{i}, v_{j} \in V$.

Given a QCN $N=(V, C)$, a consistent instantiation of $N$ over $V^{\prime} \subseteq V$ is a mapping $\alpha$ from $V^{\prime}$ to $\mathrm{D}$ such that for every pair $\left(v_{i}, v_{j}\right) \in V^{\prime} \times V^{\prime}$, $\left(\alpha\left(v_{i}\right), \alpha\left(v_{j}\right)\right)$ satisfies $N[i, j]$, i.e., there exists a basic relation $b \in N[i, j]$ such that $\left(\alpha\left(v_{i}\right), \alpha\left(v_{j}\right)\right) \in b$ for every $v_{i}, v_{j} \in V^{\prime}$. A solution of $N$ is a consistent instantiation of $N$ over $V$. $N$ is consistent iff it admits a solution. A sub-network $N^{\prime}$ of $N$ is a QCN $\left(V, C^{\prime}\right)$ such that $N^{\prime}[i, j] \subseteq N[i, j]$, for every pair of variables $v_{i}, v_{j}$. A scenario $\sigma$ is a QCN such that each constraint is defined by a singleton relation of $2^{\mathrm{B}}$, i.e., a relation containing exactly one basic relation. Let $\sigma$ be a scenario, the basic relation specifying the constraint between two variables $v_{i}$ and $v_{j}$ is denoted by $\sigma_{i j}$. A scenario $\sigma$ of $N$ is a sub-network of $N$. In the rest of this paper, $\langle N\rangle$ denotes the set of scenarios of $N$ and $[N]$ the set of its consistent scenarios. Two QCNs $N$ and $N^{\prime}$ are said to be equivalent, denoted by $N \equiv N^{\prime}$, iff $[N]=\left[N^{\prime}\right] . N_{A l l}^{V}$ denotes the QCN on $V$ such that for each pair of variables $\left(v_{i}, v_{j}\right), N_{A l l}^{V}[i, j]=\{e q\}$ if $v_{i}=v_{j}, N_{A l l}^{V}[i, j]=$ B otherwise. $N_{A l l}^{V}$ represents the complete lack of information about the relative positions of the variables.

Figures 2(a), 2(b) and 2(c) represent respectively a QCN $N$ of Interval Algebra defined on the set $V=\left\{v_{1}, v_{2}, v_{3}, v_{4}\right\}$, an inconsistent scenario $\sigma$ of $N$ and a consistent scenario $\sigma^{\prime}$ of $N$. A solution $\alpha$ of $\sigma^{\prime}$ is represented on Figure $2(\mathrm{~d})$. In order to alleviate the figures, for each pair of variables $\left(v_{i}, v_{j}\right)$, we do not represent the constraint $N[i, j]$ when $N[i, j]=\mathrm{B}$, when $N[j, i]$ is represented or when $i=j$.

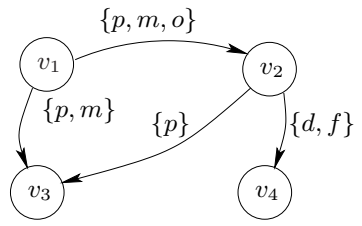

(a) $N$

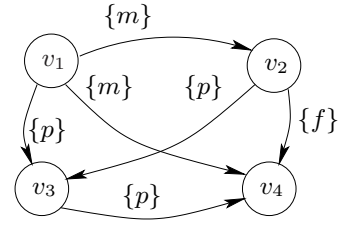

(b) $\sigma$

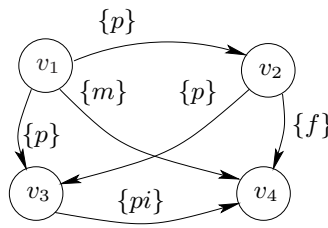

(c) $\sigma^{\prime}$

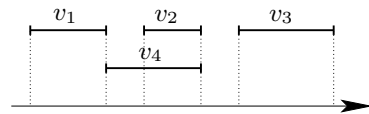

(d) $\alpha$

Fig. 2. A QCN $N$, an inconsistent scenario $\sigma$ of $N$, a consistent scenario $\sigma^{\prime}$ of $N$ and a solution of $\sigma^{\prime}$. 


\subsection{Basic distances and aggregation functions.}

In the following, we consider two classes of QCN merging operators parameterized by a distance between basic relations of $\mathrm{B}$ called basic distance, and by aggregation functions.

Basic distances. A basic distance associates with a pair of basic relations of $\mathrm{B}$ a positive number representing their degree of closeness [6].

Definition 2 (Basic distance). A basic distance $d_{\mathrm{B}}$ is a pseudo-distance, i.e., a mapping from $\mathrm{B} \times \mathrm{B}$ to $\mathbb{R}_{0}^{+}$such that $\forall b, b^{\prime} \in \mathrm{B}$, we have:

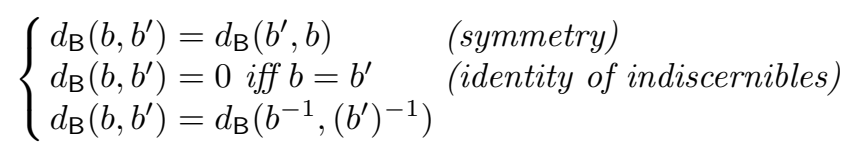

For instance, the drastic distance $d_{D}$ is equal to 1 for every pair of distinct basic relations, 0 otherwise.

In the context of qualitative algebras, two distinct basic relations can be more or less close from each other. This intuition takes its source in works of Freksa [7] who defined different notions of conceptual neighborhood between basic relations of Interval Algebra. By generalizing his definition, it is natural to state that two basic relations $b, b^{\prime} \in \mathrm{B}$ are conceptually neighbors if a continuous transformation on the elements of the domain leads to two entities which satisfy the basic relation $b$ and also directly satisfy the basic relation $b^{\prime}$ without satisfying any other basic relation. A conceptual neighborhood defines a binary relation on elements of $B$. This relation can be represented by an undirected connected graph in which every vertice is an element of B. In such a graph, called conceptual neighborhood graph, two vertices connected by an edge are conceptual neighbors. For example, in a context where a continuous transformation between two intervals corresponds to moving only one of the four possible bounds, we get the conceptual neighborhood graph $\mathrm{GB}_{\text {int }}$ depicted in Figure 3.

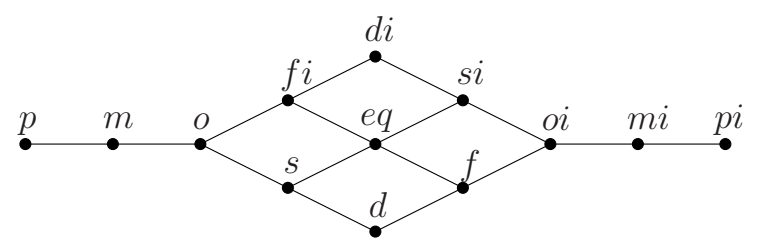

Fig. 3. The conceptual neighborhood graph $\mathrm{GB}_{\text {int }}$ of Interval Algebra.

Using conceptual neighborhood graphs a specific basic distance has been defined in the context of QCNs in [6]. The so-called conceptual neighborhood distance is formally defined as follows: 
Definition 3 (Conceptual neighborhood distance). Let GB be a conceptual neighborhood graph on $\mathrm{B}$. The conceptual neighborhood distance $d_{\mathrm{GB}}(a, b)$ between two basic relations $a, b \in \mathrm{B}$ is the length of the shortest chain leading from a to $b$ in $\mathrm{GB}$.

In the following examples, we will use the conceptual neighborhood distance $d_{\mathrm{GB}_{\text {int }}}$ defined from the graph $\mathrm{GB}_{\text {int }}$. For instance, $d_{\mathrm{GB}_{\text {int }}}(m, d i)=4$. Notice that $d_{\mathrm{GB}_{\text {int }}}$ is a basic distance in the sense of Definition 2.

Aggregation functions. An aggregation function $[18,11,12]$ typically combines in a given manner several numerical values into a single one.

Definition 4 (Aggregation function). An aggregation function $f$ associates with a vector of non-negative real numbers a non-negative real number verifying the following properties:

$\begin{cases}\text { if } x_{1} \leq x_{1}^{\prime}, \ldots, x_{p} \leq x_{p}^{\prime}, \text { then } f\left(x_{1}, \ldots, x_{p}\right) \leq f\left(x_{1}^{\prime}, \ldots, x_{p}^{\prime}\right) & \text { (non-decreasingness) } \\ x_{1}=\cdots=x_{p}=0 \text { iff } f\left(x_{1}, \ldots, x_{p}\right)=0 & \text { (minimality) }\end{cases}$

Many aggregation functions have been considered so far in various contexts. For instance, $\sum$ (sum), Max (maximum), Leximax ${ }^{1}$ are often considered in the belief merging setting $[17,21,13,11,14]$. We give some additional properties on aggregation functions.

Definition 5 (Properties on aggregation functions). Let $f$ and $g$ be two aggregation functions.

- $f$ is symmetric iff for every permutation $\tau$ from $\mathbb{R}_{0}^{p}$ to $\mathbb{R}_{0}^{p}, p$ being a strictly positive integer, $f\left(x_{1}, \ldots, x_{p}\right)=f\left(\tau\left(x_{1}\right), \ldots, \tau\left(x_{p}\right)\right)$.

- $f$ is associative iff $f\left(f\left(x_{1}, \ldots, x_{p}\right), f\left(y_{1}, \ldots, y_{p^{\prime}}\right)\right)=f\left(x_{1}, \ldots, x_{p}, y_{1}, \ldots, y_{p^{\prime}}\right)$.

- $f$ is strictly non-decreasing iff if $x_{1} \leq x_{1}^{\prime}, \ldots, x_{p} \leq x_{p}^{\prime}$ and $\exists i \in\{1, \ldots, p\}, x_{i}<$ $x_{i}^{\prime}$, then $f\left(x_{1}, \ldots, x_{p}\right)<f\left(x_{1}^{\prime}, \ldots, x_{p}^{\prime}\right)$.

- $f$ commutes with $g$ (or $f$ and $g$ are commuting aggregation functions) iff $f\left(g\left(x_{1,1}, \ldots, x_{1, q}\right), \ldots, g\left(x_{p, 1}, \ldots, x_{p, q}\right)\right)=g\left(f\left(x_{1,1}, \ldots, x_{p, 1}\right), \ldots\right.$,

$$
\left.f\left(x_{1, q}, \ldots, x_{p, q}\right)\right) \text {. }
$$

For example, the aggregation function $\sum$ is symmetric and associative, hence it commutes with itself, as well as the aggregation function Max. Symmetry means that the order of the aggregated values does not affect the result, associativity means that the aggregation of values can be factorized into partial aggregations. In the rest of this paper, aggregation functions will be supposed to be symmetric, i.e., they aggregate multi-sets of numbers instead of vectors of numbers. In [22], the authors focus on commuting aggregation functions since such functions play a significant role in any two-step merging process for which the result should not depend on the order of the aggregation processes. In the end of Section 4.2 we stress the influence of commuting aggregation functions in our merging procedures.

\footnotetext{
${ }^{1}$ the Leximax function induces an ordering on vectors of real numbers sorted decreasingly using the standard lexicographic ordering on those vectors, but it can be seen as an aggregation function in the sense of Definition 4 (see [11], Definition 5.1)
} 


\section{The Merging Issue}

\subsection{Problem and example}

Let $V=\left\{v_{1}, \ldots, v_{n}\right\}$ be a set of variables and $\mathcal{N}=\left\{N^{1}, \ldots, N^{m}\right\}$ be a multiset of QCNs defined over $V . \mathcal{N}$ is called a profile. Every input QCN $N^{k} \in \mathcal{N}$ stems from a particular agent $k$ providing her own preferences or beliefs about the relative configurations over $V$. Every constraint $N^{k}[i, j]$ corresponds to the set of basic relations that agent $k$ considers as possibly satisfied by $\left(v_{i}, v_{j}\right)$. In such a setting, two kinds of inconsistency are likely to appear. On the one hand, a QCN $N^{k}$ may be inconsistent since the agent expresses local preferences over pairs of variables. Therefore an inconsistency may arise without the agent being necessarily aware of that. On the other hand, the multiplicity of sources makes that the underlying QCNs are generally conflicting when combined. For example, in case of preferences representation, a single conflict of interest between two agents about the same pair of variables is sufficient to introduce inconsistency.

Consider a group of three students expressing their preferences about the schedule of fours common courses: Operating Systems (OS), Algebra, Analysis and Programming. Every student of the group provides a set of binary relations between these courses. The variables we consider here are four temporal entities $v_{1}, v_{2}, v_{3}, v_{4}$ that respectively correspond to OS, Algebra, Analysis, Programming and that form the set $V$. We consider Interval Algebra to model qualitative relations between these courses. For exemple, the first student prefers to start learning OS before the beginning of Algebra and to finish studying OS before the end of Algebra. This can be expressed by the relation $v_{1}\{p, m, o\} v_{2}$. The three students provide the QCNs $N^{1}, N^{2}, N^{3}$ depicted in Figure 4 and forming the profile $\mathcal{N}$. Notice that the conflict occurring in the example sketched in the introduction is represented in the QCN $N^{3}$, indeed there does not exist any consistent instantiation of $N^{3}$ over $\left\{v_{2}, v_{3}, v_{4}\right\}$.

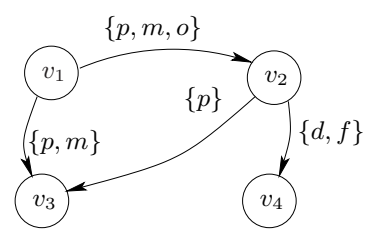

(a) $N^{1}$

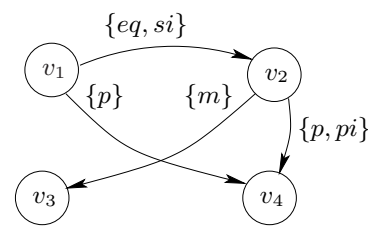

(b) $N^{2}$

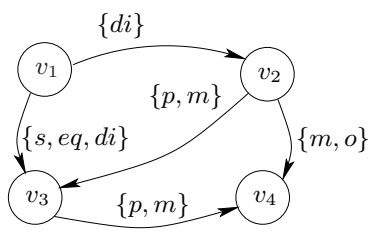

(c) $N^{3}$

Fig. 4. Three QCN $N^{1}, N^{2}$ and $N^{3}$ to be merged.

\subsection{Rationality postulates for QCN merging operators}

Given a profile $\mathcal{N}=\left\{N^{1}, \ldots, N^{m}\right\}$ defined on $V$ representing local preferences or beliefs of a set of agents, we want to get as result of the merging operation a non-empty set of consistent information representing $\mathcal{N}$ in a global way. 
Formally, this calls for a notion of merging operator. In [5] a set of rationality postulates has been proposed for QCN merging operators. These postulates are the direct counterparts in the QCN setting of the postulates from [13] characterizing merging operators for propositional logic. We separate these postulates into two classes: the first one defines the QCN merging operators, the second one provides additional properties that QCN merging operators should satisfy to exhibit a rational behaviour.

Definition 6 (QCN merging operator). An operator $\Delta$ is a mapping which associates with a profile $\mathcal{N}$ a set $\Delta(\mathcal{N})$ of consistent scenarios. Let $\mathcal{N}$ be a profile. $\Delta$ is a QCN merging operator iff it satisfies the following postulates:

$(N 1) \Delta(\mathcal{N}) \neq \emptyset$.

(N2) If $\bigcap\left\{\left[N^{k}\right] \mid N^{k} \in \mathcal{N}\right\} \neq \emptyset$, then $\Delta(\mathcal{N})=\bigcap\left\{\left[N^{k}\right] \mid N^{k} \in \mathcal{N}\right\}$.

(N1) ensures that the result of the merging is non-trivial; (N2) requires $\Delta(\mathcal{N})$ to be the set of consistent scenarios shared by all $N^{k} \in \mathcal{N}$, when this set is nonempty.

Before giving the additional postulates, we need to define the notion of equivalence between profiles. Two profiles $\mathcal{N}$ and $\mathcal{N}^{\prime}$ are said to be equivalent, denoted by $\mathcal{N} \equiv \mathcal{N}^{\prime}$, iff there exists a one-to-one correspondence $f$ between $\mathcal{N}$ and $\mathcal{N}^{\prime}$ such that $\forall N^{k} \in \mathcal{N}, f\left(N^{k}\right) \equiv N^{k}$. We use $\sqcup$ to denote the union operator for multisets.

Definition 7 (postulates (N3) - (N6)). Let $\mathcal{N}, \mathcal{N}_{1}$ and $\mathcal{N}_{2}$ be three profiles, and let $N, N^{\prime}$ be two consistent $\mathrm{QCNs}$.

(N3) If $\mathcal{N}_{1} \equiv \mathcal{N}_{2}$, then $\Delta\left(\mathcal{N}_{1}\right)=\Delta\left(\mathcal{N}_{2}\right)$.

(N4) If $\Delta\left(\left\{N, N^{\prime}\right\}\right) \cap[N] \neq \emptyset$, then $\Delta\left(\left\{N, N^{\prime}\right\}\right) \cap\left[N^{\prime}\right] \neq \emptyset$.

(N5) $\Delta\left(\mathcal{N}_{1}\right) \cap \Delta\left(\mathcal{N}_{2}\right) \subseteq \Delta\left(\mathcal{N}_{1} \sqcup \mathcal{N}_{2}\right)$.

(N6) If $\Delta\left(\mathcal{N}_{1}\right) \cap \Delta\left(\mathcal{N}_{2}\right) \neq \emptyset$, then $\Delta\left(\mathcal{N}_{1} \sqcup \mathcal{N}_{2}\right) \subseteq \Delta\left(\mathcal{N}_{1}\right) \cap \Delta\left(\mathcal{N}_{2}\right)$.

(N3) is the syntax-irrelevance principle for QCNs. It states that if two profiles are equivalent, then merging independently each profile should lead to the same result. (N4) is an equity postulate, it requires the QCN merging operator not to exploit any hidden preference between two QCNs to be merged. (N5) and (N6) together ensure that when merging independently two profiles leads both results to share a non-empty set of consistent scenarios, let us say $E$, then merging the joint profiles should return $E$ as result.

\section{Two classes of QCN merging operators}

In this section, we define two classes of QCN merging operators. Operators from the first and second class are respectively denoted by $\Delta_{1}$ and $\Delta_{2}$. These operators associate with a profile $\mathcal{N}$ a set of consistent scenarios that are the "closest" ones to $\mathcal{N}$ in terms of "distance". The difference between $\Delta_{1}$ and $\Delta_{2}$ is inherent to the definition of such a distance. 
For $i \in\{1,2\}$, a QCN merging operator $\Delta_{i}$ is characterized by a triple $\left(d_{\mathrm{B}}, f_{i}, g_{i}\right)$ where $d_{\mathrm{B}}$ is a basic distance on $\mathrm{B}$ and $f_{i}$ and $g_{i}$ are two associative and symmetric aggregation functions. $\Delta_{i}$ is then denoted by $\Delta_{i}^{d_{\mathrm{B}}, f_{i}, g_{i}}$. The set of consistent scenarios $\Delta_{i}^{d_{\mathrm{B}}, f_{i}, g_{i}}(\mathcal{N})$ is the result of a two-step process.

\section{1 $\quad \Delta_{1}$ operators}

The first step consists in computing a local distance $d_{f_{1}}$ between every consistent scenario on $V$, i.e., every element of $\left[N_{A l l}^{V}\right]$ and each QCN of the profile $\mathcal{N}$. For this purpose, the basic distance $d_{\mathrm{B}}$ and the aggregation function $f_{1}$ are used to define the distance $d_{f_{1}}$ between two scenarios $\sigma$ and $\sigma^{\prime}$ of $N_{A l l}^{V}$, as follows:

$$
d_{f_{1}}\left(\sigma, \sigma^{\prime}\right)=f_{1}\left\{d_{\mathrm{B}}\left(\sigma_{i j}, \sigma_{i j}^{\prime}\right) \mid v_{i}, v_{j} \in V, i<j\right\} .
$$

Therefore the distance between two scenarios results from the aggregation of distances at the constraints level. The definition of $d_{f_{1}}$ is extended in order to compute a distance between a consistent scenario $\sigma$ of $N_{A l l}^{V}$ and a QCN $N^{k}$ of $\mathcal{N}$ as follows:

$$
d_{f_{1}}\left(\sigma, N^{k}\right)=\min \left\{d_{f_{1}}\left(\sigma, \sigma^{\prime}\right) \mid \sigma^{\prime} \in\left\langle N^{k}\right\rangle\right\} .
$$

Therefore the distance between a scenario $\sigma$ and a QCN $N^{k}$ is the minimal distance (w.r.t. $d_{f_{1}}$ ) between $\sigma$ and a scenario of $N^{k}$.

The choice of the aggregation function $f_{1}$ depends on the context. For example, $f_{1}=$ Max is appropriate when only the greatest distance over all constraints between a scenario and a QCN is important, whatever their number. However, by instantiating $f_{1}=\sum$, the distances $d_{\mathrm{B}}$ over all constraints are summed up, thus all of them are taken into account.

Example (continued). For the sake of conciseness, we represent a scenario as the list of its constraints following the lexicographical order over $\left(v_{i}, v_{j}\right), i<j$. For instance, the consistent scenario $\sigma_{1}$ depicted in Figure $5(\mathrm{a})$ is specified by the list $(\{f i\},\{m\},\{p\},\{m\},\{p\},\{m\})$. Let $\sigma^{\prime \prime}$ be the (inconsistent) scenario of $N^{1}$ (see Figure 4(a)) defined by $(\{o\},\{m\},\{p\},\{p\},\{d\},\{m\})$. We use here the basic distance $d_{\mathrm{GB}_{\text {int }}}$ and will do so for the next examples. We consider $f_{1}=\sum$. Then we have:

$$
\begin{aligned}
d_{\sum}\left(\sigma_{1}, N^{1}\right)= & \min \left\{d_{\sum}\left(\sigma_{1}, \sigma^{\prime}\right) \mid \sigma^{\prime} \in\left\langle N^{1}\right\rangle\right\}=d_{\sum}\left(\sigma_{1}, \sigma^{\prime \prime}\right) \\
= & \sum\left\{d_{\mathrm{GB}_{\text {int }}}(f i, o), d_{\mathrm{GB}_{\text {int }}}(m, m), d_{\mathrm{GB}_{\text {int }}}(p, p),\right. \\
& \left.d_{\mathrm{GB}_{\text {int }}}(m, p), d_{\mathrm{GB}_{\text {int }}}(p, d), d_{\mathrm{GB}_{\text {int }}}(m, m)\right\} \\
= & 1+0+0+1+4+0=6 .
\end{aligned}
$$

Similarly we get $d_{\sum}\left(\sigma_{1}, N^{2}\right)=1$ and $d_{\sum}\left(\sigma_{1}, N^{3}\right)=4$.

The second step of the merging process consists in taking advantage of the aggregation function $g_{1}$ to aggregate the local distances $d_{f_{1}}\left(\sigma, N^{k}\right)$ for every QCN $N^{k} \in \mathcal{N}$; the resulting value can be viewed as a global distance $d_{g_{1}}$ between $\sigma$ and the profile $\mathcal{N}$. This distance is defined as follows:

$$
d_{g_{1}}(\sigma, \mathcal{N})=g_{1}\left\{d_{f_{1}}\left(\sigma, N^{k}\right) \mid N^{k} \in \mathcal{N}\right\}
$$




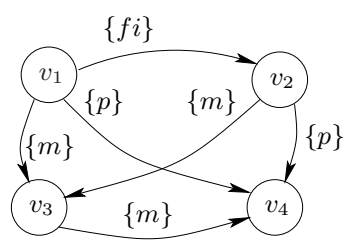

(a) $\sigma_{1}$

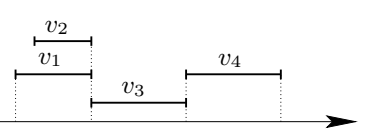

(b) $\alpha_{1}$

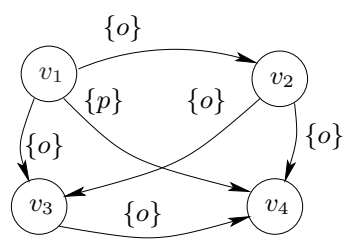

(c) $\sigma_{2}$

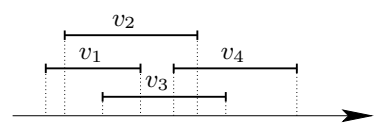

(d) $\alpha_{2}$

Fig. 5. Two consistent scenarios $\sigma_{1}$ and $\sigma_{2}$ of $N_{A l l}^{V}$, and two consistent instantiations $\alpha_{1}$ and $\alpha_{2}$ of $\sigma_{1}$ and $\sigma_{2}$.

For the arbitration function $g_{1}=\operatorname{Max}$, the global distance represents a consensual value w.r.t. all sources [21]; with $g_{1}=\sum$, it reflects the majority point of view of the sources [17].

Example (continued). Consider here $g_{1}=$ Max. We have :

$$
d_{\text {Max }}\left(\sigma_{1}, \mathcal{N}\right)=\max \left\{d_{\sum}\left(\sigma_{1}, N^{k}\right) \mid N^{k} \in \mathcal{N}\right\}=\max \{6,1,4\}=6 .
$$

The set $\Delta_{1}^{d_{\mathrm{B}}, f_{1}, g_{1}}(\mathcal{N})$ is the set of the consistent scenarios of $N_{A l l}^{V}$ having a minimal global distance $d_{g_{1}}$. Formally,

$$
\Delta_{1}^{d_{\mathrm{B}}, f_{1}, g_{1}}(\mathcal{N})=\left\{\sigma \in\left[N_{A l l}^{V}\right] \mid \nexists \sigma^{\prime} \in\left[N_{A l l}^{V}\right], d_{g_{1}}\left(\sigma^{\prime}, \mathcal{N}\right)<d_{g_{1}}(\sigma, \mathcal{N})\right\} .
$$

Example (continued). Consider the consistent scenario $\sigma_{2}$ depicted in Figure $5(\mathrm{c})$. We can compute its global distance similarly as for $\sigma_{1}$. We then have $d_{\text {Max }}\left(\sigma_{2}, \mathcal{N}\right)=5$. Since $d_{\operatorname{Max}}\left(\sigma_{2}, \mathcal{N}\right)<d_{\operatorname{Max}}\left(\sigma_{1}, \mathcal{N}\right)$, we can conclude that the consistent scenario $\sigma_{1}$ does not belong to the set $\Delta_{1}^{d_{\mathrm{GB} \text { int }}, \sum, \operatorname{Max}}(\mathcal{N})$.

Proposition 1. $\Delta_{1}^{d_{\mathrm{B}}, f_{1}, g_{1}}$ is a QCN merging operator in the sense of Definition 6, i.e., it satisfies postulates (N1) and (N2). Moreover, if $g_{1}$ is an associative aggregation function, then $\Delta_{1}^{d_{\mathrm{B}}, f_{1}, g_{1}}$ satisfies (N5), and if $g_{1}$ is an associative and strictly non-decreasing aggregation function, then $\Delta_{1}^{d_{\mathrm{B}}, f_{1}, g_{1}}$ satisfies (N6). It does not satisfy (N3) and (N4).

\section{$4.2 \quad \Delta_{2}$ operators}

An operator from the $\Delta_{2}$ family is defined in two steps as follows. The first step consists in computing a local distance $d_{f_{2}}$ between every basic relation of $\mathrm{B}$ and 
the multiset $\mathcal{N}[i, j]=\left\{N^{k}[i, j] \mid N^{k} \in \mathcal{N}\right\}$, for every pair $\left(v_{i}, v_{j}\right), i<j$. The definition of the basic distance $d_{\mathrm{B}}$ between two basic relations of $\mathrm{B}$ is extended to the basic distance between a basic relation $b \in \mathrm{B}$ and a relation $R \in 2^{\mathrm{B}}, R \neq \emptyset$. It corresponds to the minimal basic distance between $b$ and every basic relation of $R$. Formally we write:

$$
d_{\mathrm{B}}(b, R)=\min \left\{d_{\mathrm{B}}\left(b, b^{\prime}\right) \mid b^{\prime} \in R\right\} .
$$

The aggregation function $f_{2}$ is used to compute the local distance between every basic relation of B and the multiset of constraints $\mathcal{N}[i, j]=\left\{N^{k}[i, j] \mid N^{k} \in\right.$ $\mathcal{N}\}$ as follows:

$$
d_{f_{2}}(b, \mathcal{N}[i, j])=f_{2}\left\{d_{\mathrm{B}}\left(b, N^{k}[i, j]\right) \mid N^{k}[i, j] \in \mathcal{N}[i, j]\right\} .
$$

The choice of $f_{2}$ is motivated in the same way as that of $g_{1}$ for $\Delta_{1}$ operators. Depending on the context, we opt for a majority function $\sum$ [17], or for an arbitration function $\operatorname{Max}$ [21]. Here the aggregation step relates the constraints $N^{k}[i, j]$ of the QCNs $N^{k} \in \mathcal{N}$, for a given pair of variables $\left(v_{i}, v_{j}\right), i<j$.

Example (continued). Consider the multiset $\mathcal{N}[1,2]=\{\{p, m, o\},\{e q, s i\},\{d i\}\}$ (see Figure 4). We consider $d_{\mathrm{GB}_{\text {int }}}$ as the basic distance and $f_{2}=$ Max. The distance between the basic relation $f i$ and the multiset $\mathcal{N}[1,2]$ is defined as follows:

$$
\begin{aligned}
d_{\text {Max }}(f i, \mathcal{N}[1,2])= & \max \left\{d_{\mathrm{GB}_{\text {int }}}(f i,\{p, m, o\}),\right. \\
& \left.d_{\mathrm{GB}_{\text {int }}}(f i,\{e q, s i\}), d_{\mathrm{GB}_{\text {int }}}(f i,\{d i\})\right\} \\
= & \max \left\{d_{\mathrm{GB}_{\text {Bint }}}(f i, o), d_{\mathrm{GB}_{\text {int }}}(f i, e q), d_{\mathrm{GB}_{\text {int }}}(f i, d i)\right\} \\
= & \max \{1,1,1\}=1 .
\end{aligned}
$$

The second step consists in aggregating the local distances computed in the previous step for all pairs $\left(v_{i}, v_{j}\right), i<j$, in order to compute a global distance $d_{g_{2}}$ between a scenario $\sigma$ of $N_{A l l}^{V}$ and the profile $\mathcal{N}$. This distance is computed using the aggregation function $g_{2}$ as follows:

$$
d_{g_{2}}(\sigma, \mathcal{N})=g_{2}\left\{d_{f_{2}}\left(\sigma_{i j}, \mathcal{N}[i, j]\right) \mid v_{i}, v_{j} \in V, i<j\right\} .
$$

The choice of $g_{2}$ is motivated in the same way as the aggregation function $f_{1}$ for $\Delta_{1}$ operators.

Example (continued). Consider again the consistent scenario $\sigma_{1}$ (see Figure 5(a)) and choose $g_{2}=\sum$. We get:

$$
\begin{aligned}
d_{\sum}\left(\sigma_{1}, \mathcal{N}\right) & =\sum\left\{d_{\operatorname{Max}}\left(\sigma_{1}(1,2), \mathcal{N}[1,2]\right), \ldots, d_{\operatorname{Max}}\left(\sigma_{1}(3,4), \mathcal{N}[3,4]\right)\right\} \\
& =1+2+0+1+4+0=8 .
\end{aligned}
$$

Similarly to $\Delta_{1}$ operators, the result of the merging process over the profile $\mathcal{N}$ using $\Delta_{2}^{d_{\mathrm{B}}, f_{2}, g_{2}}$ corresponds to the set of consistent scenarios of $N_{A l l}^{V}$ that minimize the global distance $d_{g_{2}}$. Formally,

$$
\Delta_{2}^{d_{B}, f_{2}, g_{2}}(\mathcal{N})=\left\{\sigma \in\left[N_{A l l}^{V}\right] \mid \nexists \sigma^{\prime} \in\left[N_{A l l}^{V}\right], d_{g_{2}}\left(\sigma^{\prime}, \mathcal{N}\right)<d_{g_{2}}(\sigma, \mathcal{N})\right\} .
$$


Example (continued). Consider again the consistent scenario $\sigma_{2}$ depicted in Figure $5(\mathrm{c})$. Its global distance to $\mathcal{N}$, computed similarly to the one of $\sigma_{1}$, is $d_{\sum}\left(\sigma_{2}, \mathcal{N}\right)=8$. Notice that the consistent scenarios $\sigma_{1}$ and $\sigma_{2}$ have the same global distance to $\mathcal{N}$. We can then conclude that $\sigma_{1} \in \Delta_{2}^{d_{\mathrm{GB} \text { int }}, \operatorname{Max}, \Sigma}(\mathcal{N})$ iff $\sigma_{2} \in \Delta_{2}^{d_{\mathrm{GB}_{\text {int }}}, \operatorname{Max}, \Sigma}(\mathcal{N})$.

One can prove that $\Delta_{2}$ operators typically satisfies less expected postulates than the $\Delta_{1}$ ones:

Proposition 2. $\Delta_{2}^{d_{\mathrm{B}}, f_{2}, g_{2}}$ is a QCN merging operator in the sense of Definition 6, i.e., it satisfies the postulates (N1) and (N2). The postulates (N3) - (N6) are not satisfied.

That $\Delta_{1}$ and $\Delta_{2}$ are syntactical operators is reflected by the fact that they do not satisfy the syntax-independence postulate (N3) (see Propositions 1 and 2). Similarly in [10] several syntax-sensitive propositional merging operators have been investigated, none of them satisfying the counterpart of (N3) in the propositional setting. We give some conditions under which $\Delta_{1}$ and $\Delta_{2}$ operators are equivalent.

Proposition 3. If $f_{1}=g_{2}, f_{2}=g_{1}$ and $f_{1}$ and $f_{2}$ are commuting aggregation functions, then $\Delta_{1}^{d_{\mathrm{B}}, f_{1}, g_{1}}(\mathcal{N})=\Delta_{2}^{d_{\mathrm{B}}, f_{2}, g_{2}}(\mathcal{N})$.

Consequently, when $f_{1}=g_{2}, f_{2}=g_{1}$ and for instance when $\left(f_{1}, f_{2}\right) \in$ $\left\{\left(\sum, \sum\right),(\operatorname{Max}, \operatorname{Max})\right\}$, then choosing a $\Delta_{1}$ operator rather than a $\Delta_{2}$ one (or conversely) has no impact on the result. However, $\sum$ and Max are not commuting aggregation functions, so for such choices using $\Delta_{1}$ or $\Delta_{2}$ can lead to different results.

\subsection{Computational complexity}

Beyond logical postulates, complexity considerations can be used as choice criteria for a QCN merging operator. Clearly enough, the merging result may be of exponential size in the worst case, just like representation of the merging result in the propositional case $[3,11,12]$. As it has been already discussed in [6], a set of consistent scenarios can not always be represented in a compact way by a QCN. In [6] a basic construction of a QCN $N_{S}$ is given from a set $S$ of consistent scenarios leading to $S=\left[N_{S}\right]$ when it is possible, $S \subset\left[N_{S}\right]$ otherwise. Nevertheless, computing explicitly the merging result (as a set of consistent scenarios in our setting, as a propositional formula in the propositional framework) is not mandatory to reason with $[3,11,12]$; often it is enough to be able to determine whether a given scenario belongs to it. This is why we focus on the following MEMBERSHIP problem (MS for short): given $i \in\{1,2\}, d_{\mathrm{B}}$ a basic distance, $f_{i}, g_{i}$ two aggregation functions, $\mathcal{N}$ a profile and $\sigma_{*}$ a scenario, does $\sigma_{*}$ belong to $\Delta_{i}^{d_{\mathrm{B}}, f_{i}, g_{i}}(\mathcal{N})$ ? The following proposition provides an upper bound of complexity for the MS problem. 
Proposition 4. If $f_{i}, g_{i}$ are computed in polynomial time, then MS $\in$ coNP.

Interestingly, usual aggregation functions like $\sum$ or Max can be computed in polynomial time. For the merging procedure proposed in [6], MS is likely harder, i.e., falls to a complexity class above coNP in the polynomial hierarchy. Indeed for both $\Delta_{1}$ and $\Delta_{2}$ operators, the global distance between a scenario and a profile is computed in polynomial time (see the proof of Proposition 4). In comparison, for the merging operator proposed in [6], computing the global distance between a scenario and a profile requires the computation of all consistent scenarios of every QCN of the profile, which are exponentially many in the worst case.

\section{Comparison between $\Delta_{1}, \Delta_{2}$ and related works}

\subsection{When to choose a $\Delta_{1}$ operator}

Given a profile $\mathcal{N}$, opting for an operator $\Delta_{1}$ is appropriate when the sources are independent of one another, i.e., when information provided by each QCN of the profile should be treated independently. Indeed the first aggregation step is "local" to a particular QCN, while the second one is an "inter-source" aggregation. In this respect, $\Delta_{1}$ operators are close to QCN merging operators $\Theta$ proposed in [6] and propositional merging operators $\mathbf{D A}^{2}$ studied in $[11,12]$. In [6] the QCN merging operators $\Theta$ consider like $\Delta_{1}$ operators a profile $\mathcal{N}$ as input and return a set of consistent scenarios following a similar two-step process, with only $f_{1}=\sum$. However, while $\Delta_{1}$ operators consider the sets of scenarios of the QCNs of $\mathcal{N}$ in the computation of the local distance $d_{f_{1}}, \Theta$ operators consider the sets of their consistent scenarios. Doing so, neither inconsistent QCNs of $\mathcal{N}$ are taken into account by $\Theta$ operators, nor the basic relations of the constraints of the QCNs which do not participate in any consistent scenario of this QCN. In $[11,12]$ the authors define a class $\mathbf{D} \mathbf{A}^{2}$ of propositional knowledge bases merging operators, based on a distance between interpretations and two aggregation functions. A profile corresponds in this case to a multiset of knowledge bases, each one expressed as a finite set of propositional formulas. A first step consists in computing a local distance between an interpretation $\omega$ and a knowledge base $K$ through the aggregation of the distances between $\omega$ and every propositional formula of $K$. A second step then consists in aggregating the local distances to combine all knowledge bases of the profile. In the context of QCN merging, the $\Delta_{1}$ operators typically follow the same merging principle.

\subsection{When to choose a $\Delta_{2}$ operator}

$\Delta_{2}$ operators are suited to the context when a global decision should be made a priori for every pair of variables $\left(v_{i}, v_{j}\right)$. In this case every pair of variables is considered as a "criterion" or "topic" on which a mutual agreement has to be found as a first step. The second step then can be viewed as a relaxation of the independence criteria which are combined in order to find consistent global 
configurations. $\Delta_{2}$ operators consider a local distance $d_{f_{2}}$ which coincides with the one proposed in [4]. In this work, the authors use this local distance $d_{f_{2}}$ to define a constraint merging operator. Such an operator associates with a multiset $\mathcal{R}$ of relations the set of basic relations for which the distance $d_{f_{2}}$ to $\mathcal{R}$ is minimal. In this framework, a QCN merging operator, denoted by $\Omega$, associates with a profile $\mathcal{N}$ a single QCN $\Omega(\mathcal{N})$. Similarly to $\Delta_{2}$ operators, $\Omega$ operators take into consideration inconsistent QCNs and consider every basic relation of all constraints of the input QCNs as a relevant piece of information in the merging process. However, $\Omega$ operators require to be given a fixed total ordering $<_{V}$ on the pairs of variables $\left(v_{i}, v_{j}\right)$. Following this ordering, the constraint of the QCN $\Omega(\mathcal{N})$ bearing on $\left(v_{i}, v_{j}\right)$ is affected using the constraint merging operator on the constraints of the QCNs of $\mathcal{N}$ bearing on $\left(v_{i}, v_{j}\right)$. At each step, $\Omega(\mathcal{N})$ is kept consistent. Though the computation of $\Omega(\mathcal{N})$ is efficient, the choice of $<_{V}$ leads to specific results, while $\Delta_{2}$ operators - which do not require $<_{V}$ to be specified - do not suffer from this drawback.

\section{Conclusion}

In this paper, we have defined two classes $\Delta_{1}$ and $\Delta_{2}$ of operators for merging qualitative constraint networks (QCNs) defined on the same qualitative formalism. We have studied their logical properties and we have also considered the problem of deciding whether a given scenario belongs to the result of the merging. From a methodology point of view, we have addressed the problem of choosing such a merging operator. Compared with previous merging operators, $\Delta_{1}$ and $\Delta_{2}$ operators achieve a good compromise to QCN merging. Indeed, (i) they take into account fine-grained information provided by the input sources in the sense that each constraint from the input QCNs participates in the merging process (in particular inconsistent scenarios are not excluded); (ii) the computational complexity of query answering for those operators is not very high; (iii) they are QCN merging operators since rationality postulates (N1) and (N2) hold. Interestingly, our operators do not trivialize when applied to a single inconsistent QCN; as such, they can also be viewed as consistency restoring operators.

As a matter for further research, we plan to investigate in depth the complexity issues for all classes of operators defined so far.

\section{References}

1. J-F. Allen. An interval-based representation of temporal knowledge. In Proc. of the Seventh International Joint Conference on Artificial Intelligence (IJCAI), pages 221-226, 1981.

2. P. Balbiani, J-F. Condotta, and L. Fariñas del Cerro. A new tractable subclass of the rectangle algebra. In Proc. of the Sixteenth International Joint Conference on Artificial Intelligence (IJCAI), pages 442-447, 1999.

3. M. Cadoli, F. M. Donini, P. Liberatore, and M. Schaerf. The size of a revised knowledge base. Artificial Intelligence, 115(1):25-64, 1999. 
4. J-F. Condotta, S. Kaci, P. Marquis, and N. Schwind. Merging qualitative constraint networks in a piecewise fashion. In Proc. of the Twenty-First International Conference on Tools with Artificial Intelligence (ICTAI), pages 605-608, 2009.

5. J-F. Condotta, S. Kaci, P. Marquis, and N. Schwind. Merging qualitative constraints networks using propositional logic. In Proc. of the Tenth European Conference on Symbolic and Quantitative Approaches to Reasoning with Uncertainty (ECSQARU), pages 347-358, 2009.

6. J-F. Condotta, S. Kaci, and N. Schwind. A Framework for Merging Qualitative Constraints Networks. In Proc. of the Twenty-First International Florida Artificial Intelligence Research Society Conference (FLAIRS), pages 586-591, 2008.

7. Christian Freksa. Temporal reasoning based on semi-intervals. Artificial Intelligence, 54(1):199-227, 1992.

8. A. Gerevini and J. Renz. Combining topological and size information for spatial reasoning. Artificial Intelligence, 137(1-2):1-42, 2002.

9. S. Kaci and C. Piette. Looking for the best and the worst. In Colloque sur l'Optimisation et les Systèmes d'Information (COSI), 2009.

10. S. Konieczny. On the difference between merging knowledge bases and combining them. In Proc. of the Seventh International Conference on Principles of Knowledge Representation and Reasoning (KR), pages 135-144, 2000.

11. S. Konieczny, J. Lang, and P. Marquis. Distance-based merging: a general framework and some complexity results. In Proc. of the Eighth International Conference on Principles of Knowledge Representation and Reasoning (KR), pages 97-108, 2002.

12. S. Konieczny, J. Lang, and P. Marquis. $\mathbf{D A}^{2}$ merging operators. Artificial Intelligence, 157(1-2):49-79, 2004.

13. S. Konieczny and R. Pino Pérez. On the logic of merging. In Proc. of the Sixth International Conference on Principles of Knowledge Representation and Reasoning (KR), pages 488-498, 1998.

14. S. Konieczny and R. Pino Prez. Merging information under constraints: a logical framework. Journal of Logic and Computation, 12(5):773-808, 2002.

15. G. Ligozat. Reasoning about cardinal directions. Journal of Visual Languages and Computing, 9(1):23-44, 1998.

16. G. Ligozat and J. Renz. What Is a Qualitative Calculus? A General Framework. In Proc. of the Eighth Pacific Rim International Conference on Artificial Intelligence (PRICAI), pages 53-64, 2004.

17. J. Lin. Integration of weighted knowledge bases. Artificial Intelligence, 83(2):363378, 1996.

18. J.-L. Marichal. Aggregation Operators for Multicriteria Decision Aid. PhD thesis, Institute of Mathematics, University of Liège, Liège, Belgium, 1998.

19. D-A. Randell, Z. Cui, and A. Cohn. A spatial logic based on regions and connection. In Proc. of the Third International Conference on Principles of Knowledge Representation and Reasoning (KR), pages 165-176. 1992.

20. J. Renz and D. Mitra. Qualitative direction calculi with arbitrary granularity. In Proc. of the Ninth Pacific Rim International Conference on Artificial Intelligence (PRICAI), pages 65-74, 2004.

21. P. Z. Revesz. On the Semantics of Arbitration. Journal of Algebra and Computation, 7 (2):133-160, 1997.

22. S. Saminger-Platz, R. Mesiar, and D. Dubois. Aggregation operators and commuting. IEEE T. Fuzzy Systems, 15(6):1032-1045, 2007. 
23. D. Thau, S. Bowers, and B. Ludäscher. Merging taxonomies under RCC-5 algebraic articulations. In Proc. of the Second International Workshop on Ontologies and Information Systems for the Semantic Web (ONISW), pages 47-54, 2008.

24. P. van Beek. Reasoning about qualitative temporal information. In Proc. of the Eighth National Conference on Artificial Intelligence (AAAI), pages 728-734, 1990.

\section{Appendix}

Proposition 1. $\Delta_{1}^{d_{\mathrm{B}}, f_{1}, g_{1}}$ is a QCN merging operator in the sense of Definition 6, i.e., it satisfies postulates (N1) and (N2). Moreover, $\Delta_{1}^{d_{\mathrm{B}}, f_{1}, g_{1}}$ satisfies (N5), and if $g_{1}$ is a strictly non-decreasing aggregation function, then $\Delta_{1}^{d_{\mathrm{B}}, f_{1}, g_{1}}$ satisfies (N6). It does not satisfy (N3) and (N4).

Proof. (N1) By definition.

(N2) Let $\sigma \in\left[N_{A l l}^{V}\right]$. From the identity of indiscernibles property of $d_{\mathrm{B}}$, we have $\forall \sigma^{\prime} \in\left\langle N_{A l l}^{V}\right\rangle, \forall v_{i}, v_{j}, i<j, d_{\mathrm{B}}\left(\sigma_{i j}, \sigma_{i j}^{\prime}\right)=0$ iff $\sigma_{i j}=\sigma_{i j}^{\prime}$. Hence, since $f_{1}$ satisfies the property of minimality, we have $\forall \sigma^{\prime} \in\left\langle N_{A l l}^{V}\right\rangle, d_{f_{1}}\left(\sigma, \sigma^{\prime}\right)=0$ iff $\sigma=\sigma^{\prime}$. Thus $d_{f_{1}}\left(\sigma, N^{k}\right)=0$ iff $\sigma \in\left[N^{k}\right]$. By minimality of $g_{1}$, we have $d_{g_{1}}(\sigma, \mathcal{N})=0$ iff $\sigma \in\left[N^{k}\right] \forall N^{k} \in \mathcal{N}$. Yet $\bigcap\left\{\left[N^{k}\right] \mid N^{k} \in \mathcal{N}\right\} \neq \emptyset$. Therefore by definition of $\Delta_{1}^{d_{\mathrm{B}}, f_{1}, g_{1}}, \sigma \in \Delta_{1}^{d_{\mathrm{B}}, f_{1}, g_{1}}(\mathcal{N})$ iff $\sigma \in \bigcap\left\{\left[N^{k}\right] \mid N^{k} \in \mathcal{N}\right\}$.

(N5) Let $\mathcal{N}_{1}$ and $\mathcal{N}_{2}$ be two profiles. Let $\sigma \in \Delta_{1}^{d_{\mathrm{B}}, f_{1}, g_{1}}\left(\mathcal{N}_{1}\right) \cap \Delta_{1}^{d_{\mathrm{B}}, f_{1}, g_{1}}\left(\mathcal{N}_{2}\right)$ and let $\sigma^{\prime} \in\left[N_{A l l}^{V}\right]$. We have $d_{g_{1}}\left(\sigma, \mathcal{N}_{1}\right) \leq d_{g_{1}}\left(\sigma^{\prime}, \mathcal{N}_{1}\right)$ and $d_{g_{1}}\left(\sigma, \mathcal{N}_{2}\right) \leq d_{g_{1}}\left(\sigma^{\prime}, \mathcal{N}_{2}\right)$, or still $g_{1}\left\{d_{f_{1}}\left(\sigma, N^{k}\right) \mid N^{k} \in \mathcal{N}_{1}\right\} \leq g_{1}\left\{d_{f_{1}}\left(\sigma^{\prime}, N^{k}\right) \mid N^{k} \in \mathcal{N}_{1}\right\}$ and $g_{1}\left\{d_{f_{1}}\left(\sigma, N^{k}\right) \mid\right.$ $\left.N^{k} \in \mathcal{N}_{2}\right\} \leq g_{1}\left\{d_{f_{1}}\left(\sigma^{\prime}, N^{k}\right) \mid N^{k} \in \mathcal{N}_{2}\right\}$. Hence, by associativity and nondecreasingness of $g_{1}$, we have $g_{1}\left\{d_{f_{1}}\left(\sigma, N^{k}\right) \mid N^{k} \in \mathcal{N}_{1} \sqcup \mathcal{N}_{2}\right\} \leq g_{1}\left\{d_{f_{1}}\left(\sigma^{\prime}, N^{k}\right) \mid N^{k} \in\right.$ $\left.\mathcal{N}_{1} \sqcup \mathcal{N}_{2}\right\}$. Therefore $\sigma \in \Delta_{1}^{d_{\mathrm{B}}, f_{1}, g_{1}}\left(\mathcal{N}_{1} \sqcup \mathcal{N}_{2}\right)$.

(N6) Let $\mathcal{N}_{1}$ and $\mathcal{N}_{2}$ be two profiles. Let $\sigma \in \Delta_{1}^{d_{\mathrm{B}}, f_{1}, g_{1}}\left(\mathcal{N}_{1} \sqcup \mathcal{N}_{2}\right)$. By absurd, let us suppose that $\sigma \notin \Delta_{1}^{d_{\mathrm{B}}, f_{1}, g_{1}}\left(\mathcal{N}_{1}\right) \cap \Delta_{1}^{d_{\mathrm{B}}, f_{1}, g_{1}}\left(\mathcal{N}_{2}\right)$. Let $\sigma \notin \Delta_{1}^{d_{\mathrm{B}}, f_{1}, g_{1}}\left(\mathcal{N}_{1}\right)$ (the proof is similar if we suppose $\left.\sigma \notin \Delta_{1}^{d_{\mathrm{B}}, f_{1}, g_{1}}\left(\mathcal{N}_{2}\right)\right)$. Since $\Delta_{1}^{d_{\mathrm{B}}, f_{1}, g_{1}}\left(\mathcal{N}_{1}\right) \cap \Delta_{1}^{d_{\mathrm{B}}, f_{1}, g_{1}}\left(\mathcal{N}_{2}\right) \neq$ $\emptyset, \exists \sigma^{\prime} \in\left[N_{A l l}^{V}\right]$ such that $d_{g_{1}}\left(\sigma^{\prime}, \mathcal{N}_{1}\right)<d_{g_{1}}\left(\sigma, \mathcal{N}_{1}\right)$ and $d_{g_{1}}\left(\sigma^{\prime}, \mathcal{N}_{2}\right) \leq d_{g_{1}}\left(\sigma, \mathcal{N}_{2}\right)$, or still $g_{1}\left\{d_{f_{1}}\left(\sigma^{\prime}, N^{k}\right) \mid N^{k} \in \mathcal{N}_{1}\right\}<g_{1}\left\{d_{f_{1}}\left(\sigma, N^{k}\right) \mid N^{k} \in \mathcal{N}_{1}\right\}$ and $g_{1}\left\{d_{f_{1}}\left(\sigma^{\prime}, N^{k}\right) \mid N^{k} \in \mathcal{N}_{2}\right\} \leq g_{1}\left\{d_{f_{1}}\left(\sigma, N^{k}\right) \mid N^{k} \in \mathcal{N}_{2}\right\}$. Hence, by associativity and strict non-decreasingness of $g_{1}$, we have $g_{1}\left\{d_{f_{1}}\left(\sigma^{\prime}, N^{k}\right) \mid N^{k} \in \mathcal{N}_{1} \sqcup \mathcal{N}_{2}\right\}<$ $g_{1}\left\{d_{f_{1}}\left(\sigma, N^{k}\right) \mid N^{k} \in \mathcal{N}_{1} \sqcup \mathcal{N}_{2}\right\}$, that contradicts $\sigma \in \Delta_{1}^{d_{\mathrm{B}}, f_{1}, g_{1}}\left(\mathcal{N}_{1} \sqcup \mathcal{N}_{2}\right)$.

Proposition 2. $\Delta_{2}^{d_{\mathrm{B}}, f_{2}, g_{2}}$ is a QCN merging operator in the sense of Definition 6, i.e., it satisfies the postulates (N1) and (N2). The postulates (N3) - (N6) are not satisfied.

Proof. (N1) By definition.

(N2) From the indentity of indiscernibles property of $d_{\mathrm{B}}$, we have $\forall b \in \mathrm{B}, \forall R \in$ $2^{\mathrm{B}}, d_{\mathrm{B}}(b, R)=0$ iff $b \in R$. Let $\mathcal{R}$ be a multiset of relations of $2^{\mathrm{B}}$. By minimality of $f_{2}$, we have $d_{f_{2}}(b, \mathcal{R})=0$ iff $b \in R \forall R \in \mathcal{R}$. Let $\sigma \in\left[N_{A l l}^{V}\right]$. By minimality of $g_{2}$, we have $d_{g_{2}}(\sigma, \mathcal{N})=0$ iff $\forall v_{i}, v_{j}, i<j, \sigma_{i j} \in N^{k}[i, j] \forall N^{k} \in \mathcal{N}$. This means $\sigma \in \Delta_{2}^{d_{\mathrm{B}}, f_{2}, g_{2}}(\mathcal{N})$ iff $\sigma \in \bigcap\left\{\left[N^{k}\right] \mid N^{k} \in \mathcal{N}\right\}$. 
Proposition 3. If $f_{1}=g_{2}, f_{2}=g_{1}$ and $f_{1}$ and $f_{2}$ are commuting aggregation functions, then $\Delta_{1}^{d_{\mathrm{B}}, f_{1}, g_{1}}(\mathcal{N})=\Delta_{2}^{d_{\mathrm{B}}, f_{2}, g_{2}}(\mathcal{N})$.

Proof. It is sufficient to show that global distances $d_{g_{1}}$ and $d_{g_{2}}$ (respectively steping in the computation of $\Delta_{1}^{d_{\mathrm{B}}, f_{1}, g_{1}}(\mathcal{N})$ and $\left.\Delta_{2}^{d_{\mathrm{B}}, f_{2}, g_{2}}(\mathcal{N})\right)$ coincide. Let $N^{k} \in$ $\mathcal{N}$ and $\sigma \in\left[N_{A l l}^{V}\right]$. Let $h$ be an aggregation function, let us denote $\alpha^{k}(h)=$ $d_{h}\left(\sigma, N^{k}\right)=\min \left\{h\left\{d_{\mathrm{B}}\left(\sigma_{i j}, \sigma_{i j}^{\prime}\right) \mid v_{i}, v_{j} \in V, i<j\right\} \mid \sigma^{\prime} \in\left\langle N^{k}\right\rangle\right\}$ and $\beta^{k}(h)=$ $h\left\{\min \left\{d_{\mathrm{B}}\left(\sigma_{i j}, b\right) \mid b \in N^{k}[i, j]\right\} \mid v_{i}, v_{j} \in V, i<j\right\}$. Let us show first that $\alpha^{k}\left(f_{1}\right)=\beta^{k}\left(f_{1}\right)$. By non-decreasingness of $f_{1}$, we clearly have $\alpha^{k}\left(f_{1}\right) \geq \beta^{k}\left(f_{1}\right)$, thus let us show $\alpha^{k}\left(f_{1}\right) \leq \beta^{k}\left(f_{1}\right)$. Let $\sigma^{\prime}$ be a consistent scenario defined $\forall v_{i}, v_{j}$ by $\sigma_{i j}^{\prime}=b$ such that $b \in N^{k}[i, j]$ and $d_{\mathrm{B}}\left(\sigma_{i j}, b\right)=\min \left\{d_{\mathrm{B}}\left(\sigma_{i j}, b^{\prime}\right) \mid b^{\prime} \in N^{k}[i, j]\right\}$. By definition, $\sigma^{\prime} \in\left\langle N^{k}\right\rangle$, hence $\beta^{k}\left(f_{1}\right)=f_{1}\left\{d_{\mathrm{B}}\left(\sigma_{i j}, \sigma_{i j}^{\prime}\right) \mid v_{i}, v_{j} \in V, i<j\right\}$. Therefore $\alpha^{k}\left(f_{1}\right) \leq \beta^{k}\left(f_{1}\right)$. The global distance $d_{g_{1}}$ between $\sigma$ and $\mathcal{N}$ steping in the computation of $\Delta_{1}^{d_{\mathrm{B}}, f_{1}, g_{1}}(\mathcal{N})$ is defined as $d_{g_{1}}(\sigma, \mathcal{N})=g_{1}\left\{\alpha^{k}\left(f_{1}\right) \mid N^{k} \in\right.$ $\mathcal{N}\}=g_{1}\left\{\beta^{k}\left(f_{1}\right) \mid N^{k} \in \mathcal{N}\right\}$. Since $f_{1}=g_{2}$ and $f_{2}=g_{1}$, we have $d_{g_{1}}(\sigma, \mathcal{N})=$ $f_{2}\left\{\beta^{k}\left(g_{2}\right) \mid N^{k} \in \mathcal{N}\right\} . d_{g_{2}}$ is the global distance steping in the computation of $\Delta_{1}^{d_{\mathrm{B}}, f_{2}, g_{2}}(\mathcal{N})$; since $f_{2}$ and $g_{2}$ are commuting aggregation functions, we have $d_{g_{1}}(\sigma, \mathcal{N})=d_{g_{2}}(\sigma, \mathcal{N})$.

Proposition 4. If $f_{i}, g_{i}$ are computed in polynomial time, then MS $\in$ coNP.

Proof. It is sufficient to consider the following algorithm:

(1) guess a scenario $\sigma$ over $V$;

(2) check that $\sigma$ is consistent;

(3) compute $d_{g_{i}}(\sigma, \mathcal{N})$ and $d_{g_{i}}\left(\sigma_{*}, \mathcal{N}\right)$, and check that $d_{g_{i}}(\sigma, \mathcal{N})<d_{g_{i}}\left(\sigma_{*}, \mathcal{N}\right)$;

Step (2) runs in polynomial time, for every qualitative algebra.

It can be shown that for $i \in\{1,2\}$, step (3) runs in polynomial time. For $\Delta_{2}$, it is obvious from the definition of the global distance $d_{g_{2}}$ and since $f_{2}, g_{2}$ are computed in polynomial time. For $\Delta_{1}$, as it is shown in the proof of Proposition 3 (without assuming the hypotheses of Proposition 3), we have for every scenario $\sigma$ and for every QCN $N^{k} \in \mathcal{N}, d_{f_{1}}\left(\sigma, N^{k}\right)=f_{1}\left\{\min \left\{d_{\mathrm{B}}\left(\sigma_{i j}, b\right) \mid b \in\right.\right.$ $\left.\left.N^{k}[i, j]\right\} \mid v_{i}, v_{j} \in V, i<j\right\}$ (i.e., the global distance $d_{f_{1}}\left(\sigma, N^{k}\right)$ between a scenario $\sigma$ and a QCN $N^{k}$ can be computed componentwise). Yet $\min \left\{d_{\mathrm{B}}\left(\sigma_{i j}, b\right) \mid b \in\right.$ $\left.N^{k}[i, j]\right\}$ is computed in constant time since the cardinality of $\mathrm{B}$ is a constant, and $f_{1}, g_{1}$ are computed in polynomial time. Therefore $d_{g_{1}}$ is computed in polynomial time.

Thus this algorithm runs in polynomial time and it shows that the complementary problem of MS is in NP, from which the result follows. 